\title{
Vacuum Stability in two-Higgs doublet models
}

\author{
Nuno Barros e Sá \\ DCTD, Universidade dos Açores \\ 9500-801 Ponta Delgada, Portugal \\ E-mail: nunosa@uac.pt
}

\section{Augusto Barroso}

Centro de Física Teórica e Computacional, Faculdade de Ciências, Universidade de Lisboa, Avenida Professor Gama Pinto, 2, 1649-003 Lisboa, Portugal;

E-mail: barroso@cii.fc.ul.pt

\section{Pedro Ferreira}

Instituto Superior de Engenharia de Lisboa, Rua Conselheiro Emídio Navarro 1, 1959-007

Lisboa, Portugal;

Centro de Física Teórica e Computacional, Faculdade de Ciências, Universidade de Lisboa, Avenida Professor Gama Pinto, 2, 1649-003 Lisboa, Portugal.

E-mail: ferreira@cii.fc.ul.pt

\section{Rui Santos*†}

NExT Institute and School of Physics and Astronomy, University of Southampton Highfield, Southampton SO17 1BJ, United Kingdom

E-mail: santos@pp.rhul.ac.uk

In this work we review the status of tree-level vacuum stability in general two-Higgs doublet models. We also discuss the problem of Normal minima in some classes of potentials. In some of these potentials, Normal minima can coexist leading to potentially dangerous physic scenarios as, for instance, a two step spontaneous symmetry breaking.

Prospects for Charged Higgs Discovery at Colliders

16-19 September 2008

Uppsala, Sweden

\footnotetext{
${ }^{*}$ Speaker.

†This work is supported by Fundação para a Ciência e Tecnologia under contract PTDC/FIS/70156/2006. R.S. is supported by the FP7 via a Marie Curie Intra European Fellowship, contract number PIEF-GA-2008-221707.
} 


\section{Introduction}

The Standard Model (SM) is a theory invariant under the symmetry group $S U(3)_{c} \otimes S U(2)_{L} \otimes$ $U(1)_{Y}$. In the SM, the spontaneous symmetry breaking (SSB) mechanism is accomplished with one scalar doublet whose vacuum expectation value (vev) is usually written as $\left\langle\phi_{S M}\right\rangle=\left(\begin{array}{lll}0 & v / \sqrt{2}\end{array}\right)$ where $v=246 \mathrm{GeV}$. The charge operator is defined as $Q=1 / 2\left(\sigma_{3}+Y I\right)$, where $\sigma_{3}$ is the standard Pauli matrix, $I$ is the $2 \times 2$ identity matrix and $Y$ is the weak hypercharge. With this definition $Q<\phi_{S M}>=0$ as it should because the vacuum has no electric charge. Had we started with the most general vacuum configuration $\left\langle\phi_{S M}\right\rangle=\left(\begin{array}{ll}v_{1}+i v_{2} & v_{3}+i v_{4}\end{array}\right) / \sqrt{2}$ we would still have a massless photon. The electromagnetic $U(1)_{e m}$ can not be broken with only one doublet and to choose the most general vacuum configuration simply amounts to a redefinition of the charge operator.

The situation changes radically once one adds a second doublet. We have now eight fields that can acquire a vev. We can however use the $S U(2)_{L} \otimes U(1)_{Y}$ gauge freedom to write the most general vacuum structure as

$$
\left(\begin{array}{c}
0 \\
v_{1} e^{i \theta_{1}}
\end{array}\right) \quad\left(\begin{array}{c}
v_{2} \\
v_{3}
\end{array}\right) \text {. }
$$

The gauge boson's mass matrix can now have four non-zero eigenvalues. The mass eigenvalue related to the photon is given by

$$
m_{\gamma}^{2}=\frac{1}{8}\left[v^{2}\left(g^{2}+g^{\prime 2}\right)-\sqrt{v^{4}\left(g^{2}+g^{\prime 2}\right)-16 g^{2} g^{\prime 2} v_{1}^{2} v_{2}^{2}}\right]
$$

where $v=\sqrt{v_{1}^{2}+v_{2}^{2}+v_{3}^{2}}$ and $g$ and $g^{\prime}$ are the $S U(2)$ and $U(1)$ gauge couplings respectively. There are two ways to recover a massless photon: either by setting $v_{1}=0$ (the SM case) or by choosing $v_{2}=0$ and the vevs are then aligned [1]. Otherwise the photon becomes massive as a consequence of the charged vacuum configuration.

Gauge invariance disallows not only a charge breaking vacuum but also a CP breaking vacuum in the SM. Again the situation changes in THDM. Defining a CP transformation as $\phi_{i} \rightarrow \phi_{i}^{*}$, three different types of vacua can be defined for a general THDM with all constants real,

$$
<\phi_{1}>_{N}=\left(\begin{array}{c}
0 \\
v_{1}
\end{array}\right) \quad<\phi_{2}>_{N}=\left(\begin{array}{c}
0 \\
v_{2}
\end{array}\right),
$$

which we call the normal vacuum,

$$
<\phi_{1}>_{C B}=\left(\begin{array}{c}
0 \\
v_{1}^{\prime}
\end{array}\right) \quad<\phi_{2}>_{C B}=\left(\begin{array}{c}
\alpha \\
v_{2}^{\prime}
\end{array}\right),
$$

for a vacuum that breaks charge conservation, and finally

$$
<\phi_{1}>_{C P}=\left(\begin{array}{c}
0 \\
v_{1}^{\prime \prime}+i \delta
\end{array}\right) \quad<\phi_{2}>_{C P}=\left(\begin{array}{c}
0 \\
v_{2}^{\prime \prime}
\end{array}\right),
$$

for a CP breaking vacuum. Vacua with $\alpha$ and $\delta$ simultaneously non-zero are not considered because the minimisation conditions of the potential forbid them ${ }^{1}$.

\footnotetext{
${ }^{1}$ Except for a very special case in the explicit $\mathrm{CP}$ breaking potential. Even in that case, though, via a basis change, that vacuum may be reduced to one with $\alpha \neq 0$ and $\delta=0$.
} 


\section{The THDM potentials}

The vacuum structure of THDM has been the subject of many studies [1-11], while the vacuum structure of an arbitrary number of doublets was studied in $[12,13]$. The scalar sector of a THDM is built with eight independent fields, four from each doublet. The number of independent gauge invariants is however four due to the SM gauge invariance under $S U(2)_{L} \otimes U(1)_{Y}$ with four gauge generators ${ }^{2}$. Therefore, all properties of the potential can be studied in terms of four independent gauge invariants, which we choose to be $x_{1}=\left|\phi_{1}\right|^{2}, x_{2}=\left|\phi_{2}\right|^{2}, x_{3}=\operatorname{Re}\left(\phi_{1}^{\dagger} \phi_{2}\right)$ and $x_{4}=\operatorname{Im}\left(\phi_{1}^{\dagger} \phi_{2}\right)$. In terms of this basis set of gauge invariants, the most general renormalizable THDM has 14 real parameters and can be written as

$$
\begin{aligned}
V= & a_{1} x_{1}+a_{2} x_{2}+a_{3} x_{3}+a_{4} x_{4}+b_{11} x_{1}^{2}+b_{22} x_{2}^{2}+b_{33} x_{3}^{2}+b_{44} x_{4}^{2}+ \\
& b_{12} x_{1} x_{2}+b_{13} x_{1} x_{3}+b_{14} x_{1} x_{4}+b_{23} x_{2} x_{3}+b_{24} x_{2} x_{4}+b_{34} x_{3} x_{4} .
\end{aligned}
$$

Under the CP transformation of the form $\phi_{i} \rightarrow \phi_{i}^{*}, x_{1}, x_{2}$ and $x_{3}$ remain the same but $x_{4}$ switches signal. Thus, the terms of the potential which are linear in $x_{4}$ break CP explicitly. Therefore a potential for which CP is not explicitly broken cannot have terms linear in $x_{4}$ in eq. 2.1: $a_{4}=b_{14}=$ $b_{24}=b_{34}=0$. This potential has 10 free parameters and can still break CP spontaneously.

To further reduce the number of parameters while keeping the model renormalizable we can force the potential to be invariant under certain symmetries imposed to the fields. In the case of THDM the $Z_{2}$ and a softly broken global $U(1)$ symmetries lead [2] to two seven parameter models with a very different phenomenology. Recently, it was shown in [14] that these are the only two "simple" symmetries that can be imposed on THDM. In [2] an interesting relation between CP-violation, symmetries and flavour changing neutral currents (FCNC) at tree level was found. In fact, for the 10-parameter potential only one of the CP-breaking stationary conditions can be forced to have no solution. This equation can be written as

$$
b_{13}\left(v_{1}^{\prime \prime 2}+\delta^{2}\right)+b_{23} v_{2}^{\prime \prime 2}+\left(b_{33}-b_{44}\right) v_{1}^{\prime \prime} v_{2}^{\prime \prime}=a_{3}
$$

and renormalizability forces $b_{13}$ and $b_{23}$ to be simultaneously either zero or non-zero. Therefore the equation has no solution in the two following situations

$$
\begin{aligned}
& b_{13}=b_{23}=a_{3}=0 ; b_{33} \neq b_{44} \quad\left(\phi \rightarrow-\phi_{1} ; \quad \phi_{2} \rightarrow \phi_{2}\right) \\
& b_{13}=b_{23}=b_{33}-b_{44}=0 ; a_{3} \neq 0 \quad\left(\phi \rightarrow e^{i \theta} \phi_{1} ; \quad \phi_{2} \rightarrow \phi_{2}\right) .
\end{aligned}
$$

Hence, forcing the CP-breaking minimum not to exist leads to two phenomenologically different 7-parameter potentials. Furthermore, by extending the symmetry to the Yukawa sector one avoids the existence of FCNC at tree level. There are still two other potentials to consider: the 6-parameter potential with an axion (exact $U(1)$ symmetry) or a potential that softly breaks $Z_{2}$ which has 8 free parameters and can be written as

$$
V_{Z_{2}}^{\text {soft }}=a_{1} x_{1}+a_{2} x_{2}+a_{3} x_{3}+b_{11} x_{1}^{2}+b_{22} x_{2}^{2}+b_{33} x_{3}^{2}+b_{44} x_{4}^{2}+b_{12} x_{1} x_{2}
$$

\footnotetext{
${ }^{2}$ A similar counting for the SM with one doublet would naively lead to the conclusion that we would end up with zero gauge invariants. However this reasoning does not apply to the SM due to the existence of a little group, subgroup of the gauge group, that leaves the doublet invariant.
} 
From the phenomenological point of view all these models can be studied as a limiting case of $V_{Z_{2}}^{\text {soft }}$ in the case of normal minima. Choosing as free parameters of $V_{Z_{2}}^{\text {soft }}$ the four Higgs masses, $\tan \beta=v_{2} / v_{1}, \alpha$ (the rotation angle in the CP-even sector) and $a_{3}$, if we set $a_{3}=0$ we obtain the potential with the exact $Z_{2}$ symmetry, $V_{Z_{2}}$ and when $a_{3}=-M_{A}^{2} / \sin (2 \beta)$ we find the potential with the softly broken $U(1)$ symmetry, $V_{U(1)}^{\text {soft }}$.

\section{Minima of different nature}

Contrary to the SM, THDM can have several stationary points of different natures. The three possible types of stationary points which we called Normal (N), Charge Breaking (CB) or CPbreaking $(\mathrm{CP})$ were defined in the introduction. Therefore, one may inquire about the possibility of having two minima of different nature for the same set of parameters. This could pose a problem to THDM as we could be living in a Normal minimum and suddenly tunnel to a deeper charge breaking minimum where the photon acquires a mass. To answer this question we have to compare the vacuum energy at the different stationary points, $V_{C B}, V_{C P}$ and $V_{N}$. In [3] we found a very interesting relation between the difference of the value of the potential at a $\mathrm{CB}$ stationary point, $V_{C B}$, and the value of the potential at a normal stationary point, $V_{N}$,

$$
V_{C B}-V_{N}=\frac{M_{H^{ \pm}}^{2}}{2 v^{2}}\left[\left(v_{1}^{\prime} v_{2}-v_{2}^{\prime} v_{1}\right)^{2}+\alpha^{2} v_{1}^{2}\right]
$$

where $v^{2}=v_{1}^{2}+v_{2}^{2}$ and $M_{H^{ \pm}}^{2}$ is the value of the squared mass of the charged Higgs scalar, evaluated at the normal stationary point. The normal stationary point is a minimum if all squared scalar masses are positive. Hence, this equation tells us that if the normal stationary point is a minimum, it is definitely below the charge breaking stationary point. Furthermore, in ref. [3] we proved that in this case the charge breaking stationary point is a saddle point. The stability of the normal minimum against tunneling to a deeper charge breaking stationary point is thus ensured in THDM.

A similar result holds when one compares a $\mathrm{CP}$ and a normal stationary point. When $\mathrm{CP}$ is a good quantum number at the potential level, we found that the difference between the value of the potential at the $\mathrm{CP}$ stationary point, $V_{C P}$, and at the normal stationary point, $V_{N}$, is given by [3]

$$
V_{C P}-V_{N}=\frac{M_{A}^{2}}{2 v^{2}}\left[\left(v_{1}^{\prime \prime} v_{2}-v_{2}^{\prime \prime} v_{1}\right)^{2}+\delta^{2} v_{2}^{2}\right]
$$

$M_{A}^{2}$ is now the value of the squared pseudoscalar mass at the normal stationary point. Again, if the normal stationary point is a minimum, positivity of $M_{A}^{2}$ ensures that the CP stationary point is above it. Furthermore, I. Ivanov has proved [11] that in this situation, the CP stationary is a saddle point. As the CP stationary point is also uniquely determined, the stability of the normal minimum against tunneling is guaranteed. Similar results hold for the CP breaking and for the $\mathrm{CB}$ minima. If a $\mathrm{CP}$ breaking stationary point is a minimum, the competing normal and charge breaking stationary points are saddle points above it - the $\mathrm{CP}$ breaking minimum is then a global one. Although probably not of fundamental importance, the CB stationary point, when a minimum is the global one. 


\section{Normal minima}

There is still one situation that deserves our attention - the case of two simultaneous normal minima. The THDM can have at most two normal minima [11] and from last section we know that minima of different nature never coexist. However we could still have two minima that had different spontaneous symmetry breaking patterns with different masses for the gauge bosons. In the most general 14-parameter potential with explicit CP-violation the relation between two normal stationary points, $N_{1}$ and $N_{2}$ as defined in eq. (1.5) is given by

$$
V_{N_{2}}-V_{N_{1}}=\frac{1}{2}\left[\left(\frac{M_{H^{ \pm}}^{2}}{v^{2}}\right)_{N_{1}}-\left(\frac{M_{H^{ \pm}}^{2}}{v^{2}}\right)_{N_{2}}\right]\left[\left(v_{1}^{\prime \prime} v_{2}-v_{2}^{\prime \prime} v_{1}\right)^{2}+\delta^{2} v_{2}^{2}\right]
$$

In this equation we have $\left(v^{2}\right)_{N_{1}}=v_{1}^{2}+v_{2}^{2}$ and $\left(v^{2}\right)_{N_{2}}=v_{1}^{\prime \prime 2}+v_{2}^{\prime \prime 2}+\delta^{2}$, and $\left(M_{H^{ \pm}}^{2}\right)_{N_{1,2}}$ are the squared charged scalar masses at each of the $N_{1}, N_{2}$ stationary points. This interesting relation tells us that the deepest stationary point will be the one with the largest ratio between the square of the charged Higgs mass and the $v^{2}$. This means that the deeper minimum has the largest splitting between the charged Higgs mass and the "theoretical" $W$ boson mass. In a CP conserving potential, the equation is similar but with $\delta=0$ because the normal minima configuration have no phases in a $\mathrm{CP}$ conserving potential. However this expression adds very little to the problem of the competing normal minima.

We have mentioned that the $\mathrm{CB}$ and $\mathrm{CP}$ stationary points are unique since they are given by linear equations on the vevs. However, this is not true for the normal stationary points. The stationarity conditions are always a set of two coupled cubic equations which can only be solved analytically for the potential with the exact $Z_{2}$ symmetry, $V_{Z_{2}}$, where the stationarity points are again uniquely determined. Therefore the stationarity equations can only be solved numerically. The question we are addressing now is the following: is it possible to have a normal minimum for a definite set of parameters with the right gauge boson masses and have another normal minimum below it with completely different masses for the gauge bosons? And the answer to this question is yes as long as the soft breaking term is present. As an example we can have for $V_{Z_{2}}^{\text {soft a local }}$ minimum with $m_{H}=m_{H^{ \pm}}=m_{A}=300 \mathrm{GeV}, m_{h}=100 \mathrm{GeV}$ and $m_{w}=80.4 \mathrm{GeV}$ and a global minimum with $m_{H}=m_{A}=436 \mathrm{GeV} m_{H^{ \pm}}=365 \mathrm{GeV}, m_{h}=190 \mathrm{GeV}$ and $m_{w}=107.5 \mathrm{GeV}$. The vacuum energy difference between the two is $V_{G}-V_{L}=-4.2 \times 10^{8}$. Hence, tunneling effects can occur in this situation and the subject needs further and more detailed study. Note however that all potentials are well behaved for the $\mathrm{CP}$ breaking minima no matter how small the $\mathrm{CP}$ violation phase is because the CP breaking minima is uniquely determined. Finaly, only the potential with the exact $Z_{2}$ symmetry guarantees that the minimum, being a normal one, is unique. Even the competing normal stationary points with one of the vevs set to zero are above it. For a discussion on this point see [10].

\section{Conclusions}

In this section we try to sum up our present knowledge about the tree-level vacuum of THDM in the following points $[2,3,8,10,11]$ : 
- THDM bounded from below have no maxima (except for the trivial);

- THDM have at most two minima;

- Minima of different nature never coexist;

- Unlike Normal minima, CB and CP minima are uniquely determined;

- If a THDM has only one normal minimum then this is the absolute minimum - all other stationary points if they exist are saddle points and above it;

- If a THDM has a CP breaking minimum then this is the absolute minimum - all other stationary points if they exist are saddle points and above it;

- THDM with no explicit CP breaking show problems for two competing normal minima;

- The THDM with an exact $Z_{2}$ symmetry has several interesting features: the minimization equations are uniquely determined which is not true when the soft breaking symmetry term is present; being in a normal minimum automatically guarantees tree-level vacuum stability no extra conditions have to be imposed; it is the only THDM where the only masses present in all scalar couplings are the ones in the corresponding interaction vertex - again this is no longer true when the soft breaking term is present. It has however the drawback of not allowing for CP violating minima. This version of the THDM is not related to the MSSM, where the existence of the soft breaking term is mandatory to avoid the existence of an axion.

\section{References}

[1] J. L. Diaz-Cruz and A. Mendez, Nucl. Phys. B 380 (1992) 39.

[2] J. Velhinho, R. Santos and A. Barroso, Phys. Lett. B 322 (1994) 213.

[3] P. M. Ferreira, R. Santos and A. Barroso, Phys. Lett. B 603 (2004) 219, [Erratum-ibid. B 629 (2005) 114]; A. Barroso, P. M. Ferreira and R. Santos, Phys. Lett. B 632 (2006) 684.

[4] I. F. Ginzburg and M. Krawczyk, Phys. Rev. D 72 (2005) 115013.

[5] A. Barroso, P. M. Ferreira and R. Santos, Afr. J. Math. Phys. 3 (2006) 103.

[6] J. F. Gunion and H. E. Haber, Phys. Rev. D 72 (2005) 095002.

[7] M. Maniatis, A. von Manteuffel, O. Nachtmann and F. Nagel, Eur. Phys. J. C 48 (2006) 805.

[8] I. P. Ivanov, Phys. Rev. D 75 (2007) 035001, [Erratum-ibid. D 76 (2007) 039902].

[9] I. F. Ginzburg and K. A. Kanishev, Phys. Rev. D 76 (2007) 095013.

[10] A. Barroso, P. M. Ferreira and R. Santos, Phys. Lett. B 652 (2007) 181.

[11] I. P. Ivanov, Phys. Rev. D 77 (2008) 015017.

[12] A. Barroso, P. M. Ferreira, R. Santos and J. P. Silva, Phys. Rev. D 74 (2006) 085016.

[13] C. C. Nishi, Phys. Rev. D 74 (2006) 036003, [Erratum-ibid. D 76 (2007) 119901]; C. C. Nishi, Phys. Rev. D 76 (2007) 055013.

[14] P. M. Ferreira and J. P. Silva, arXiv:0809.2788 [hep-ph], to appear in PRD. 